\section{Two Solutions to the Adaptive Visual Servoing Problem}

Alessandro Astolfi, Liu Hsu, Mariana S. Netto, and Romeo Ortega

\begin{abstract}
In this paper, we present two globally convergent vision-based position controllers for a planar two-links manipulator in the so-called fixed-camera configuration, where the camera orientation and scale factor are considered unknown. This is a basic adaptive visual servoing problem whose solution was hampered by the nonlinear dependence of the system dynamics on the unknown parameters. The controller design techniques of immersion and invariance and nonlinear proportional integral (PI), recently proposed in the literature, are used to derive the smooth adaptive schemes that ensure global asymptotic regulation without overparameterization, projections, or persistency of excitation assumptions. In the case of tracking, we establish error bounds that are reduced, eventually to zero, as the speed of the reference trajectory decreases, and with improved prior knowledge on the camera scale factor, for the immersion and invariance controller, or increasing a tuning gain for the nonlinear PI. The efficacy of the approaches is shown through simulations.
\end{abstract}

Index Terms-Adaptive control, camera orientation, robot manipulator, visual servoing.

\section{INTRODUCTION}

Incorporating visual information in feedback control loops represents an attractive alternative for position and motion control of autonomous robot manipulators evolving in unstructured environments [6]. In this paper, we consider the problem of adaptive visual servoing of planar robot manipulators under a fixed-camera configuration with unknown orientation and scale factor. The control goals are to place the robot end-effector in some desired constant position, or to make it track a (slowly moving) trajectory, by using a vision system equipped with a fixed camera.

It was shown in [2] (see also [9]) that a (fixed parameter) proportional derivative (PD)-like controller ensures asymptotic set-point regulation of the full robot dynamics, in spite of the uncertainty on the orientation parameter, which should, however, not be greater than $\pi / 2$. It is well known that transient performance of PD-like schemes can be improved, particularly in tracking applications, adding an adaptation feature. The uncertainty of model parameters has been of concern since the early developments. Adaptive schemes were proposed to circumvent the performance degradation due to modeling uncertainty, particularly camera calibration [14]-[16]. However, most papers in the past only considered on-line camera calibration algorithms in an ad hoc manner. The effectiveness of the methods was often justified based on experimental verification rather than theoretical justification. Only more recent papers consider the visual servoing problem as a control-theoretic problem [2], [4], [5], [7], [8], [11]. The design of an adaptive controller

Manuscript received August 24, 2001; revised January 22, 2002. This paper was recommended for publication by Associate Editor Y. Liu and Editor S. Hutchinson upon evaluation of the reviewers' comments. The work of L. Hsu was supported in part by Conselo Nacional de Desenvolvimento Científico e Technológico (CNPq), Financiadora de Estudos e Projetos (FINEP), and Fundação de Amparo à Pesquisa do Estado do Rio de Janeiro (FAPERJ). The work of M. Netto was supported by the Brazilian foundation Coordenação de Aperfeiçoamento de Pessoal de Nível Superior (CAPES).

A. Astolfi is with the Electrical Engineering Department, Imperial College, London SW7 2BT, U.K. (e-mail: a.astolfi@ic.ac.uk).

L. Hsu is with the Electrical Engineering Department, COPPE/UFRJ, 21945-970 Rio de Janeiro, Brazil (e-mail: liu@ coep.ufrj.br).

M. Netto and R. Ortega are with the Laboratory des Signaux et Systèmes, CNRS-Supélec, 91192 Gif-sur-Yvette, France (e-mail: mariana.netto@1ss.supelec.fr; romeo.ortega@1ss.supelec.fr).

Publisher Item Identifier S 1042-296X(02)06339-5. is unfortunately complicated when the unknown parameters enter nonlinearly into the system dynamics. This fact holds in our visual servoing problem, since the camera orientation angle and the scaling factor enter nonlinearly in the control matrix, which is a scaled rotation matrix. One way to bypass the nonlinearity obstacle is to consider each entry of this matrix as independent unknown constants. This solution was adopted, e.g., in [4], [5], [7], and [8]. Obviously, this increases the number of parameters to be identified (directly or indirectly), and may lead to slower convergence intrinsic to a search in a bigger space.

In [11], a globally convergent adaptive scheme that does not require overparameterization was proposed, but it uses a switching law that has been shown to yield below-par performances.

The main contribution of this paper is the development of two new, nonoverparameterized, smooth adaptive schemes that ensure that the tracking error globally asymptotically converges to a residual set, whose size reduces to zero as the speed of the reference signal goes to zero. The controllers are derived following the control design techniques of immersion and invariance (I\&I) [1] and nonlinear PI [12], recently proposed in the literature. The I\&I controller estimates the camera orientation, but fixes the scale factor to a known constant value. It requires the prior knowledge of a lower bound on the latter parameter; if it is known, then exact asymptotic global tracking of arbitrarily fast (bounded) references is ensured. Our inability to estimate two ("nonlinearly dependent") parameters motivates us to use the "root searching" stabilization procedure proposed in [12] instead of classical identification-based schemes. Interestingly, the resulting nonlinear PI controller obviates the aforementioned prior knowledge and, furthermore, incorporates a tuning gain that allows us to reduce (possibly to zero) the size of the tracking residual set. This scheme, however, presumes that the camera orientation parameter is bounded away from the critical values of $\pm \pi / 2$. The performance of the proposed controllers is illustrated with some simulations.

\section{PROBLEM FoRMULATION}

We consider a two-degrees-of-freedom robot manipulator that evolves in a plane. The vision system consists of a TV camera of charge-coupled device (CCD) type that is fixed perpendicular to the plane where the robot evolves, providing an image of the whole robot workspace, which includes the robot end-effector and the target. The image acquired by the camera supplies a two-dimensional array of brightness values from a three-dimensional scene. This image may undergo various types of computer processing to enhance image properties and extract image features [6]. As in [2], see also [3], [4], [7]-[9], we assume that the image features are the projection into the two-dimensional image plane of three-dimensional points in the scene space. Hence, we model the action of the camera as a static mapping from the joint robot positions $q \in \mathbb{R}^{2}$ to the position (in pixels) of the robot tip in the image output, denoted $y \in \mathbb{R}^{2}$. This mapping is described by

$$
y=a e^{J \theta}\left[k(q)-\vartheta_{1}\right]+\vartheta_{2}
$$

where $\theta \in \mathbb{R}$ is the orientation of the camera with respect to the robot frame, $a \in \mathbb{R}_{+}$and $\vartheta_{1}, \vartheta_{2} \in \mathbb{R}^{2}$ denote intrinsic camera parameters: scale factor, focal length, and center offset, respectively. The vector valued function $k: \mathbb{R}^{2} \rightarrow \mathbb{R}^{2}$ defines the robot direct kinematics; also, for compactness, we use the rotation matrices

$$
J=\left[\begin{array}{cc}
0 & -1 \\
1 & 0
\end{array}\right], \quad e^{J \theta}=\left[\begin{array}{cc}
\cos (\theta) & -\sin (\theta) \\
\sin (\theta) & \cos (\theta)
\end{array}\right] .
$$


Invoking standard time-scale separation arguments, we assume an inner fast loop for the robot velocity control, and concentrate on the kinematic problem where we must generate the references for the robot velocities. The robot dynamics are then described by a simple integrator $\dot{q}=\tau$, where $\tau$ are the applied joint torques. The direct kinematics yield

$$
\dot{k}=\mathcal{J}(q) \dot{q}
$$

where $\mathcal{J}(q):=(\partial k / \partial q)(q) \in \mathbb{R}^{2 \times 2}$ is the analytic robot Jacobian. As usual [2], we assume the robot operates far away from singular configurations, hence, $\mathcal{J}(q)$ is nonsingular. Differentiating (1) and replacing the latter expression, we obtain the dynamic model of the overall system of interest

$$
\dot{y}=a e^{J \theta} u
$$

where we have introduced the input change of coordinates $u:=\mathcal{J}(q) \tau$.

Adaptive Calibration Problem: Given the vision system (2), with measurable $y$ and a bounded target trajectory $y_{*}$, with known bounded first and second-order derivatives $\dot{y}_{*}, \ddot{y}_{*}$, respectively. Find a control signal $u$, such that $y$ asymptotically tracks, as closely as possible, the reference trajectory, in spite of the lack of knowledge of $a$ and $\theta$. In particular, if $y_{*}(t) \rightarrow$ const, we would like to ensure that $y(t)$ converges to $y_{*}(t)$ from all initial conditions $y(0)$.

In spite of the simplicity of the system dynamics (2), the task is, of course, complicated by the highly nonlinear dependence on the unknown parameters. In this paper, we use the I\&I and the nonlinear PI approaches proposed in [1] and [12], respectively, to design two controllers that solve the problem. Although the present work is self contained, we refer the interested reader to these papers for further details and motivation on the I\&I and nonlinear PI methods.

\section{ADAPTIVE I\&I CONTROLLER}

The first controller requires the following. ${ }^{1}$

Assumption 1: A positive lower bound on the scale factor $a$ is known, i.e., $a \geq a_{m}>0$.

To simplify the presentation of the result, we will find it convenient to define the $2 \times 2$ constant rotation matrix

$$
R:=-\frac{a}{a_{m}} e^{-J \arccos \left(a_{m} / a\right)}
$$

and the two-dimensional linear time-invariant dynamical system

$$
\dot{w}=R w-(R+I) \dot{y}_{*} .
$$

According to Assumption $1,0<a_{m} / a \leq 1$, hence, $R$ is Hurwitz. Furthermore, as

$$
\lim _{a / a_{m} \rightarrow 1} R=-I
$$

we have that, for all initial conditions, the solutions $w(t)$ of (4) will converge to zero, if either $a / a_{m} \rightarrow 1$ or $\left\|\dot{y}_{*}(t)\right\| \rightarrow 0$.

In the proposition below, we will present an adaptive I\&I controller that ensures the tracking error asymptotically converges to $w(t)$. In words, this means that we can ensure a small tracking error if either we have a good prior estimation of $a$, or the reference trajectory is slowly varying.

\footnotetext{
${ }^{1}$ This assumption will be relaxed in Section IV.
}

Proposition 1: Consider the system (2), with Assumption 1, in closed-loop with the I\&I adaptive controller

$$
\begin{aligned}
u & =-\frac{1}{a_{m}} e^{-J\left(\hat{\theta}+1 / 2\|s\|^{2}\right)} s \\
\dot{\hat{\theta}} & =s^{\top}\left(\tilde{y}+\ddot{y}_{*}\right) \\
s & =\tilde{y}-\dot{y}_{*}
\end{aligned}
$$

where $\tilde{y}:=y-y_{*}$ is the tracking error. Then, for all initial conditions $y(0) \in \mathbb{R}^{2}, \hat{\theta}(0) \in \mathbb{R}$, and all bounded reference trajectories $y_{*}$, with bounded first and second-order derivatives $\dot{y}_{*}, \ddot{y}_{*}$, we have all trajectories of the system bounded, and the tracking error either satisfies

$$
\lim _{t \rightarrow \infty}\|\tilde{y}(t)-w(t)\|=0
$$

with $w(t)$ the solution of (4) with initial conditions $w(0)=\tilde{y}(0) \in$ $\mathbb{R}^{2}$, or eventually

$$
\lim _{t \rightarrow \infty}\|s(t)\|=0, \lim _{t \rightarrow \infty} \tilde{y}(t)=0 .
$$

In particular, if either $a_{m}=a$ or $\lim _{t \rightarrow \infty}\left\|\dot{y}_{*}(t)\right\| \rightarrow 0$, then we always have $\lim _{t \rightarrow \infty} \tilde{y}(t)=0$.

Proof: First, we observe that $\theta$ is the critical parameter needed to achieve the convergence properties of the proposition. More precisely, if $\theta$ is known, we can design a stabilizing law for (2) using the lower bound estimate ${ }^{2}$ of the uncertain parameter $a$. Indeed, the constant parameter feedback $u=-\left(1 / a_{m}\right) e^{-J \theta} s$, where $s$ is defined in (7), yields the target closed-loop dynamics

$$
\dot{\tilde{y}}=-\frac{a}{a_{m}} s-\dot{y}_{*}
$$

whose trajectories converge to zero if either $a / a_{m}=1$, or $\left\|\dot{y}_{*}(t)\right\| \rightarrow$ 0 . Therefore, following the adaptive I\&I controller procedure, we will take our control law in the form

$$
u=-\frac{1}{a_{m}} e^{-J\left(\hat{\theta}+\beta_{1}(s)\right)} s
$$

where, $\beta_{1}(s)$ is a function to be determined. For convenience, we have defined this function as explicitly dependent on $s$. Introducing the coordinate

$$
z=\hat{\theta}-\theta+\beta_{1}(s)
$$

we obtain the first error equation

$$
\dot{\tilde{y}}=-\frac{a}{a_{m}} e^{-J z} s-\dot{y}_{*}
$$

which, replacing (7), can also be written in the form

$$
\dot{\tilde{y}}=-\frac{a}{a_{m}} e^{-J z} \tilde{y}-\left(-\frac{a}{a_{m}} e^{-J z}+I\right) \dot{y}_{*} .
$$

Comparing with (3) and (4), we observe that the control objective is achieved if $z(t) \rightarrow \arccos \left(a_{m} / a\right)$, which we will establish in the sequel.

\footnotetext{
${ }^{2}$ Actually, any fixed estimate of $a$ will do the job if $\theta$ is known; as will become clear later, the lower bound is needed for the adaptive problem.
} 


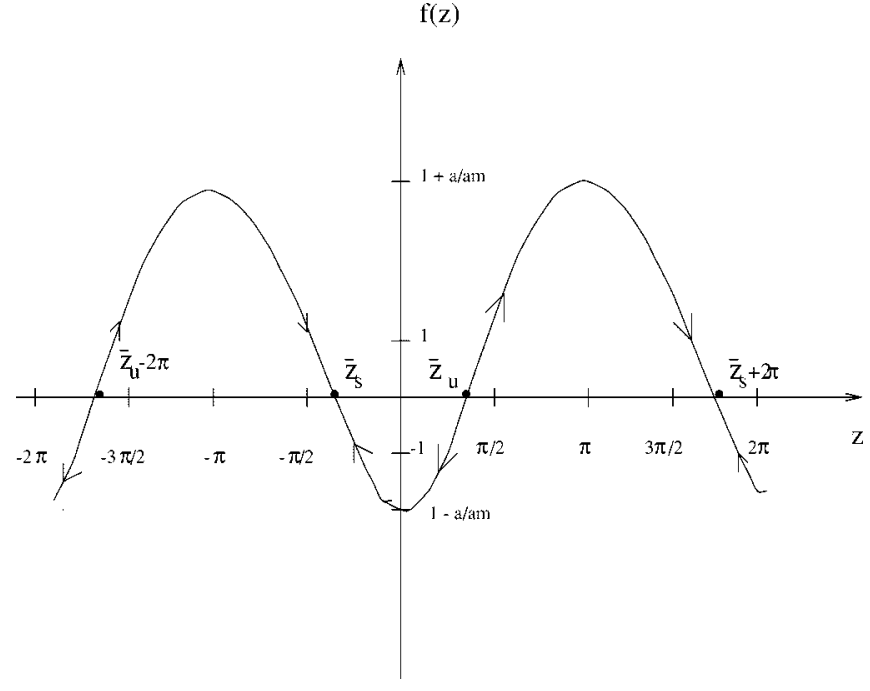

Fig. 1. Plot of the function $f(z)=1-\left(a / a_{m}\right) \cos (z)$.

The second error equation describes the dynamics of $z$, which, as seen from (11), is defined by our choice of the function $\beta_{1}(s)$ and the parameter update law. In standard applications of the I\&I approach, these two functions are selected to drive $z$ to zero. Notice that, in this case, (12) reduces the target dynamics (10). As will become clear below, due to the uncertainty in the parameter $a$, we will not obtain $z(t) \rightarrow 0$ but, as discussed in the previous paragraph, we will drive it to a value that ensures the desired stability properties. Toward this end, we select

$$
\beta_{1}(s)=\frac{1}{2}\|s\|^{2}
$$

which yields (5), and choose $\dot{\hat{\theta}}$ as given by (6). Differentiating (11), using (6) and (13), and doing some simple calculations with (7) and (12), we get the second error equation

$$
\dot{z}=-\|s\|^{2}\left[\frac{a}{a_{m}} \cos (z)-1\right]
$$

where we have used the fact that $s^{\top} e^{-J z} s=\|s\|^{2} \cos (z)$. The dynamics of the closed-loop system are fully characterized by the threedimensional error system (12), (14). To unveil the stabilization mechanism that motivated our choice for the $z$ dynamics, and explain the need for Assumption 1, we refer the reader to Fig. 1, where we plot the function $f(z)=1-\left(a / a_{m}\right) \cos (z)$. Notice that $\dot{z}=\|s\|^{2} f(z)$. Since $1-a / a_{m} \leq 0$, the function has an infinite number of roots, which define bounded intervals that are invariant to the $z$ dynamics, ensuring that, for all initial conditions, the trajectories of (14) are bounded. ${ }^{3}$ Furthermore, as nonincreasing (nondecreasing) continuous functions which are bounded from below (above) converge, we also have that $z(t) \rightarrow z_{\infty}$. As the dynamics, which are clearly $2 \pi$-periodic, exhibit alternating stable $\left(\bar{z}_{s} \bmod (2 \pi)\right)$ and unstable $\left(\bar{z}_{u} \bmod (2 \pi)\right)$ equilibria, we also conclude that, in general, $z(t) \rightarrow \bar{z}_{s} \bmod (2 \pi)$, and, in this case, $\tilde{y}$ will converge to the trajectories of (4), as desired. (Notice that $\bar{z}_{s}=\arccos \left(a_{m} / a\right)$.) The qualifier "in general" is needed because, although highly improbable, we cannot rule out the occurrence of the case $s \rightarrow 0$, hence, it needs to be considered. We will prove later that, if $s \rightarrow 0$, the tracking error actually goes to zero, which is more than expected! There also exists the possibility that $z(0)=\bar{z}_{u} \bmod (2 \pi)$,

${ }^{3}$ This property holds true independently of the behavior of the "time-scaling" factor $\|s\|^{2}$. since these are unstable equilibria, and any disturbance will drive the trajectory away from these points.

Once we have shown that $z$ is bounded, we prove now that $\tilde{y}$ is also bounded. To this end, we find it convenient to write the $\tilde{y}$ dynamics in terms of $s$ as

$$
\dot{s}=-\frac{a}{a_{m}} e^{-J z} s-\dot{y}_{*}-\ddot{y}_{*}
$$

and consider the function

$$
W(s, z)=\frac{1}{2}\|s\|^{2}-z .
$$

Taking the derivative along the trajectories of (14), (15) yields

$$
\begin{aligned}
\dot{W} & =-\|s\|^{2}-s^{\top}\left(\dot{y}_{*}+\ddot{y}_{*}\right) \\
& \leq-\frac{1}{2}\|s\|^{2}+M_{1}
\end{aligned}
$$

where $M_{1} \geq 1 / 2\left\|\dot{y}_{*}+\ddot{y}_{*}\right\|^{2}$ and we have used the inequality $2\|s\|\left\|\dot{y}_{*}+\ddot{y}_{*}\right\| \leq\|s\|^{2}+\left\|\dot{y}_{*}+\ddot{y}_{*}\right\|^{2}$. Now, from the boundedness of $z$, there exists a positive constant $c_{1}$, such that $W \leq 1 / 2\|s\|^{2}+c_{1}$. We then get the differential inequality

$$
\dot{W} \leq-W+M_{1}+c_{1}
$$

from which we immediately conclude that $W$ and, consequently, $s$ and $\tilde{y}$, are bounded.

We will now establish the convergence result (8). First, we see from (15), and the boundedness of $s$ and $\dot{y}_{*}, \ddot{y}_{*}$, that $\dot{s}$ is also bounded. Similarly, from (14) and the boundedness of $s, \dot{z}$ is bounded. Now, differentiating (14) and using the boundedness of $s, \dot{s}, \dot{z}$, we prove that $\ddot{z}$ is also bounded, hence, $\dot{z}$ is uniformly continuous. From the convergence of $z$ and Barbalat's lemma [13], we can then conclude that $\dot{z}(t) \rightarrow 0$ as $t \rightarrow \infty$. Referring to (14), this establishes that

$$
\lim _{t \rightarrow \infty}\|s(t)\|^{2}\left[1-\frac{a}{a_{m}} \cos (z(t))\right]=0
$$

which implies that either $s$, or the signal in brackets converges to zero. We consider first the case where $\lim _{t \rightarrow \infty}\left[1-\left(a / a_{m}\right) \cos (z(t))\right]=$ 0 , from which we immediately have $z(t) \rightarrow \bar{z}_{s}$. This means that the dynamics (12) can be written in the form

$$
\dot{\tilde{y}}=\left[R+B_{1}(t)\right] \tilde{y}-\left[R+B_{1}(t)+I\right] \dot{y}_{*}
$$

for some bounded matrix $\left\|B_{1}(t)\right\| \rightarrow 0$ as $t \rightarrow \infty$, and $R$ is defined in (3). The convergence result (8) then follows by subtracting (4) from the equation above.

Finally, we will prove that if ${ }^{4} \lim _{t \rightarrow \infty}\|s(t)\|=0$, then $\|\tilde{y}(t)\| \rightarrow$ 0 . From (7), we see that $\|s(t)\| \rightarrow 0$ implies that $\left\|\tilde{y}(t)-\dot{y}_{*}(t)\right\| \rightarrow 0$. On the other hand, from (12), it also implies that $\left\|\tilde{y}(t)+\dot{y}_{*}(t)\right\| \rightarrow 0$. Combining these two limits, we have $\|\dot{\tilde{y}}(t)+\tilde{y}(t)\| \rightarrow 0$, which proves that $\|\tilde{y}(t)\| \rightarrow 0$.

This completes the proof of the proposition.

\section{NONLINEAR PI CONTROLLER}

In this section, following the guidelines of [12], we design a nonlinear PI controller, where instead of estimating the parameter $\theta$ as before, we implement a "root searching" procedure for the perturbed error dynamics.

Throughout this section, we use the following.

${ }^{4}$ It is important to note that, as $s$ may converge to zero faster than $z$, we cannot conclude that $z_{\infty}=\bar{z}_{s}$. Of course, as pointed out already above, having $z_{\infty} \neq$ $\bar{z}_{\mathcal{S}}$ is highly improbable, and it is treated only for the sake of completeness. 
Assumption 2: The camera orientation $\theta$ is bounded away from $\pm \pi / 2$.

As in Proposition 1, we find it convenient to define a two-dimensional linear time-invariant "error reference" dynamics

$$
\dot{w}_{1}=R_{1} w_{1}-[\tan (\theta) J] \dot{y}_{*}
$$

where $R_{1}:=-\varepsilon[I+\tan (\theta) J]$ is a Hurwitz matrix and $\varepsilon>0$ is a tuning parameter of the controller. In the proposition below, we will present a nonlinear PI controller that ensures that the tracking error asymptotically tracks $w_{1}(t)$. To understand the implications of this property, we first observe that for all values of $\varepsilon$ and all initial conditions $w_{1}(0)$, the size of the residual set for $\left\|w_{1}(t)\right\|$ is $O\left\{\left\|\dot{y}_{*}(t)\right\|\right\}$. Hence, as for the I\&I controller, good regulation will be achieved for slowly-varying references. Furthermore, the following simple calculations prove that we can shrink the residual set by increasing $\varepsilon$. Indeed, defining $V_{w}=1 / 2\left\|w_{1}\right\|^{2}$, we can easily establish the bound

$$
\dot{V}_{w} \leq-[2 \varepsilon-\alpha] V_{w}+\frac{|\tan (\theta)|^{2}}{2 \alpha}\left\|\dot{y}_{*}\right\|^{2}
$$

where we have used the inequality

$$
w_{1}^{\top} J \dot{y}_{*} \tan (\theta) \leq \frac{\alpha}{2}\left\|w_{1}\right\|^{2}+\frac{1}{2 \alpha}|\tan (\theta)|^{2}\left\|\dot{y}_{*}\right\|^{2}
$$

which holds for all $\alpha>0$.

Proposition 2: Consider the system (2), with Assumption 2, in closed-loop with the nonlinear PI controller ${ }^{5}$

$$
\begin{aligned}
u & =-z \cos (z) s \\
z & =\hat{\theta}+\beta\left(\tilde{y}, \dot{y}_{*}\right) \\
\beta & =\frac{1}{2}\|\tilde{y}\|^{2}-\frac{1}{\varepsilon} \tilde{y}^{\top} \dot{y}_{*} \\
\dot{\hat{\theta}} & =s^{\top} \dot{y}_{*}+\frac{1}{\varepsilon} \tilde{y}^{\top} \ddot{y}_{*}+\varepsilon\|s\|^{2} \\
s & =\tilde{y}-\frac{1}{\varepsilon} \dot{y}_{*} .
\end{aligned}
$$

Then, for all initial conditions $y(0) \in \mathbb{R}^{2}, \hat{\theta}(0) \in \mathbb{R}$ and all bounded reference trajectories $y_{*}$, with bounded first and second-order derivatives $\dot{y}_{*}, \ddot{y}_{*}$, we have all trajectories of the system bounded, and the tracking error satisfies

$$
\lim _{t \rightarrow \infty}\left\|\tilde{y}(t)-w_{1}(t)\right\|=0,
$$

with $w_{1}(t)$ the solution of (17) with initial conditions $w_{1}(0)=\tilde{y}(0) \in$ $\mathbb{R}^{2}$. In particular, if either $\varepsilon \rightarrow \infty$ or $\lim _{t \rightarrow \infty}\left\|\dot{y}_{*}(t)\right\| \rightarrow 0$, then we have $\lim _{t \rightarrow \infty}\|\tilde{y}(t)\|=0$.

Proof: The first error equation is obtained by plugging (18) into (2) and using (22)

$$
\dot{\tilde{y}}=-a e^{J \theta} z \cos (z) \tilde{y}+\left(\frac{a}{\varepsilon} e^{J \theta} z \cos (z)-I\right) \dot{y}_{*} .
$$

Notice the presence of the factor $1 / \varepsilon$ in the definition of $s$.

Differentiating (19), using (21) and (20), and doing some simple calculations with (22) and (24), we get the second error equation

$$
\dot{z}=-\|s\|^{2}[(a \cos \theta) z \cos (z)-\varepsilon]
$$

where we have used the fact that $s^{\top} a e^{J \theta} s=(a \cos \theta)\|s\|^{2}$. The dynamics of the closed-loop system are fully characterized by the error system (24), and (25). (Compare with (12), and (14).)

\footnotetext{
${ }^{5}$ Both controllers are defined with the same symbols to facilitate their comparison and to establish the correspondence with the general theory developed in [1], [12]
}

Similarly to the I\&I controller, it can be directly concluded from (25) that, provided Assumption 2 is satisfied, this guarantees that $\cos \theta \neq 0, z$ is bounded, and either converges to some root $z_{\infty}$ of $(a \cos \theta) z_{\infty} \cos \left(z_{\infty}\right)-\varepsilon=0$, or $\|s(t)\| \rightarrow 0$. We prove now that $\tilde{y}$ is also bounded. To this end, we find it convenient to write the $\tilde{y}$ dynamics in terms of $s$ as

$$
\dot{s}=-a e^{J \theta} z \cos (z) s-\left(\dot{y}_{*}+\frac{1}{\varepsilon} \ddot{y}_{*}\right)
$$

and consider again the function (16), whose derivative along the trajectories of (25), and (26) yields

$$
\dot{W}=-\varepsilon\|s\|^{2}-s^{\top}\left(\dot{y}_{*}+\frac{1}{\varepsilon} \ddot{y}_{*}\right) \leq-\frac{\varepsilon}{2}\|s\|^{2}+M_{2}
$$

where $M_{2} \geq 1 /(2 \varepsilon)\left\|\dot{y}_{*}+(1 / \varepsilon) \ddot{y}_{*}\right\|^{2}$, and we have used the inequality

$$
\|s\|\left\|\dot{y}_{*}+\frac{1}{\varepsilon} \ddot{y}_{*}\right\| \leq \frac{\varepsilon}{2}\|s\|^{2}+\frac{1}{2 \varepsilon}\left\|\dot{y}_{*}+\frac{1}{\varepsilon} \ddot{y}_{*}\right\|^{2}
$$

which holds for arbitrary $\varepsilon>0$. From the fact that $z$ is bounded, we can, as in the proof of Proposition 1, establish that

$$
\dot{W} \leq-\varepsilon W+\varepsilon M_{2}+c_{2}
$$

for some $c_{2}>0$, from which we immediately conclude that $W$ and, consequently, $s$ and $\tilde{y}$, are bounded.

The proof of convergence (23) exactly mimics the one given in Proposition 1, hence it is omitted for brevity.

Before closing this section, we remark that the scheme (18)-(22) is called a nonlinear PI, because it can be written in the form

$$
\begin{aligned}
& u=\gamma\left[\beta\left(y, y_{*}\right)+\hat{\theta}, y, y_{*}\right] \\
& \dot{\hat{\theta}}=\rho\left(y, y_{*}\right)
\end{aligned}
$$

for some suitably defined proportional and integral gain functions $\beta, \rho$ and controller structure function $\gamma$. Clearly, the classical linear PI scheme is recovered, choosing the linear functions

$$
\begin{aligned}
\beta & =-K_{P}\left(y-y_{*}\right) \\
\rho & =-K_{I}\left(y-y_{*}\right) \\
\gamma & =\beta+\hat{\theta}
\end{aligned}
$$

where the constant matrices $K_{P}, K_{I}$ are the proportional and integral tuning gains. For (18)-(22) we define

$$
\begin{aligned}
\beta & =\frac{1}{2}\|\tilde{y}\|^{2}-\frac{1}{\varepsilon} \tilde{y}^{\top} \dot{y}_{*} \\
\rho & =\left(\tilde{y}-\frac{1}{\varepsilon} \dot{y}_{*}\right)^{\top} \dot{y}_{*}+\frac{1}{\varepsilon} \tilde{y}^{\top} \ddot{y}_{*}+\varepsilon\left\|\tilde{y}-\frac{1}{\varepsilon} \dot{y}_{*}\right\|^{2} \\
\gamma & =-(\hat{\theta}+\beta) \cos (\hat{\theta}+\beta)\left(\tilde{y}-\frac{1}{\varepsilon} \dot{y}_{*}\right) .
\end{aligned}
$$

\section{Simulations}

The I\&I and the nonlinear PI adaptive controllers given by (5)-(7) and (18)-(22) were tested through simulations. The two-link robot direct kinematics transformation is given by

$$
\begin{aligned}
& x_{1}=L_{1} \cos \left(q_{1}\right)+L_{2} \cos \left(q_{1}+q_{2}\right)+O_{1} \\
& x_{2}=L_{1} \sin \left(q_{1}\right)+L_{2} \sin \left(q_{1}+q_{2}\right)+O_{2}
\end{aligned}
$$

where $x_{1}$ and $x_{2}$ are the end-effector cartesian coordinates, $L_{1}$ and $L_{2}$ are the link lengths, and $x_{1 b}=O_{1}, x_{2 b}=O_{2}$ are the 

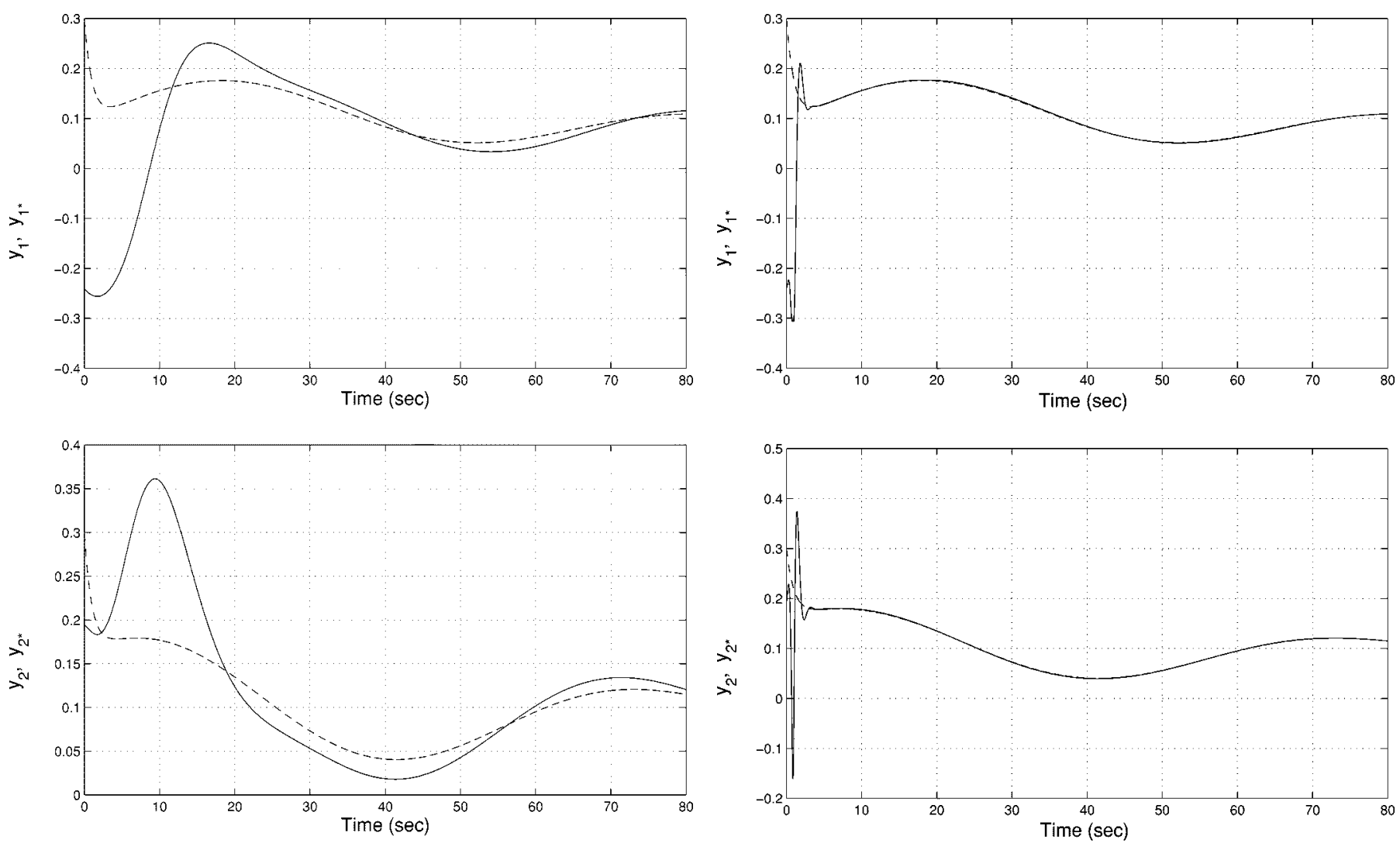

Fig. 2. Nonlinear PI controller. Behavior of the normalized image output signals $y_{1}(t)$ and $y_{2}(t)$ (full lines) and references $y_{1 *}(t)$ and $y_{2 *}(t)$ (dashed lines) for $\epsilon=0.5$

robot base coordinates in the workspace frame. For the sake of comparison, the simulations were carried out in the same conditions of [7], where $L_{1}=0.8 \mathrm{~m}, L_{2}=0.5 \mathrm{~m}, O_{1}=-0.666 \mathrm{~m}$, and $\mathrm{O}_{2}=-0.333 \mathrm{~m}$. For simplicity, the image coordinates (originally in pixels) are considered to have been normalized, i.e., the $y_{1}$ and $y_{2}$ are nondimensional.

A case of extreme misorientation was taken into consideration: $\theta=$ $1 \mathrm{rad}$, with $\hat{\theta}(0)=0$. The scaling factor $a$ and its lower bound $a_{m}$ were chosen as $a=0.7, a_{m}=0.5$. The initial conditions of the manipulator are $q_{1}(0)=1.3 \mathrm{rad}$ and $q_{2}(0)=-1.3 \mathrm{rad}$.

For the set-point control case, with $y_{1 *}=y_{2 *}=0.1$, the convergence is observed in 6 and $25 \mathrm{~s}$ for the I\&I controller and the nonlinear PI controller with $\epsilon=3$, respectively (within $4 \%$ of the final value). The control torques in the case of the I\&I controller are not greater than $0.9 \mathrm{~N} \cdot \mathrm{m}$ and in the case of the nonlinear PI, they are much smaller, not exceeding $0.17 \mathrm{~N} \cdot \mathrm{m}$.

The tracking case was also tested where the reference trajectory was generated by a first-order filter $\dot{y}_{*}=-\lambda y_{*}+r$ with the reference signals

$$
\begin{aligned}
& r_{1}=a_{1} \sin \left(w_{r} t\right)+c+d \sin \left(1.5 w_{r} t\right) \\
& r_{2}=a_{2} \sin \left(w_{r} t+\psi\right)+c+d \sin \left(1.5 w_{r} t+\psi\right)
\end{aligned}
$$

and $a_{1}=a_{2}=d=0.04, c=0.1, \psi=1 \mathrm{rad}$, and $\lambda=1$, with $w_{r}$ assuming two different values, i.e., $w_{r}=0.03$ and $w_{r}=0.07$.

The simulations were carried out for $80 \mathrm{~s}$. Different situations were performed. We first simulated the nonlinear PI controller with $w_{r}=0.07$ for $\epsilon=0.5$ (Fig. 2) and $\epsilon=20$ (Fig. 3). Observing these graphs, it becomes clear that by augmenting the parameter $\epsilon$, the tracking can be much improved. The price to be paid is the increased control effort. Nonetheless, it is lower (in absolute value)

Fig. 3. Nonlinear PI controller. Behavior of the normalized image output signals $y_{1}(t)$ and $y_{2}(t)$ (full lines) and references $y_{1 *}(t)$ and $y_{2 *}(t)$ (dashed lines) for $\epsilon=20$.
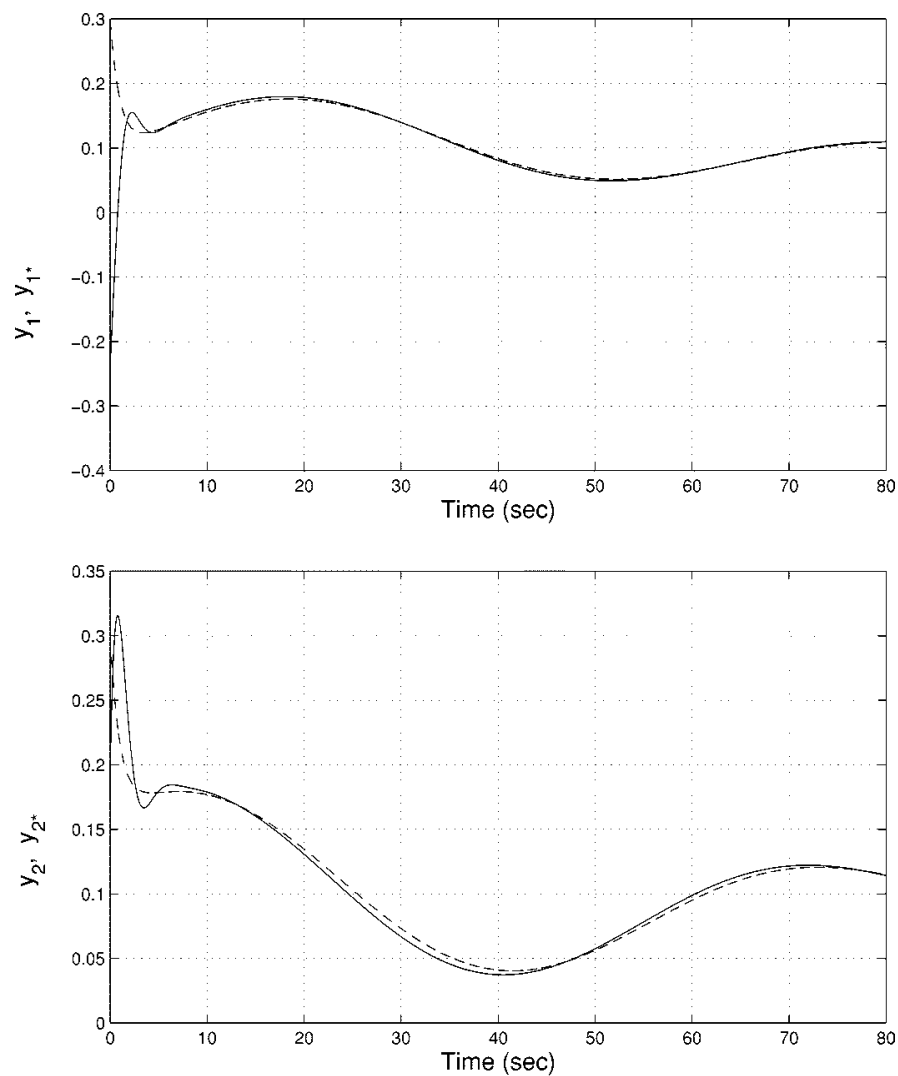

Fig. 4. I\&I adaptive controller. Behavior of the normalized image output signals $y_{1}(t)$ and $y_{2}(t)$ (full lines) and references $y_{1 *}(t)$ and $y_{2 *}(t)$ (dashed lines). 
than $5 \mathrm{~N} \cdot \mathrm{m}$ for $\epsilon=20$, whereas for $\epsilon=0.5$, it is lower than $0.17 \mathrm{~N} \cdot \mathrm{m}$. As a high gain effect, an overshoot can be noticed for the case $\epsilon=20$. It should be remarked that high controller gains require large system bandwidth to be effective. So, in practice, the controller gain is limited by the bandwidth of the vision system, usually not higher than $30 \mathrm{~Hz}$.

The I\&I controller was also tested (Fig. 4) for $w_{r}=0.07$. We can see that the tracking is still good, with a small steady-state error. As expected, if we reduce the speed of the reference trajectory (that is, $w_{r}=0.03$ ), the tracking error becomes very small. The control effort for the I\&I controller is not higher than $1.1 \mathrm{~N} \cdot \mathrm{m}$ for both reference trajectories. This is expected, since no high gain is necessary.

\section{CONCLUSIONS}

We have presented two solutions to the well-known problem of adaptive visual servoing for planar two-link manipulators when the orientation and the scale factor of the camera are unknown. Instrumental for the establishment of our results has been the utilization of the I\&I and the nonlinear PI approaches of [1] and [12], respectively.

We summarize the properties of the controllers as follows.

1) Both schemes provide a complete solution to the regulation problem, but the I\&I scheme requires a lower bound on the scale factor (Assumption 1). This stems from the fact that we could apply I\&I to adapt the orientation parameter, but have been unable to estimate the scaling factor that had to be fixed to a constant value. On the other hand, the nonlinear PI solution is applicable only to the case where the camera orientation $\theta$ is bounded away from $\pm \pi / 2$ (Assumption 2).

2) For tracking tasks, the size of the residual set for the error reduces for slowly time-varying references in both controllers. However, while on the I\&I scheme this set can only be reduced with improved prior knowledge of the scale factor $a$, the nonlinear PI controller contains a tuning parameter $\varepsilon$ that effectively regulates its size.

3) The I\&I adaptive scheme is parameter estimation-based, hence, it copies the structure of the known parameter controller and replaces the unknown parameter, in this case $\theta$, with the sum of its estimate and the function $\beta_{1}$, see (5), and (13). The nonlinear PI scheme, on the other hand, replaces this "controller structure" by a root-searching function, in this case, $z \cos (z)$.

4) From the technical viewpoint, Assumption 1 is needed to ensure the existence of equilibria for the $z$ dynamics (14). The nonlinear PI scheme obviates this prior knowledge, thanks to the utilization of the increasing function $z \cos (z)$ in (25). Although it may be argued that the design is fragile because of the possibility of injecting high gains, it is shown in [12] that our nonlinear PI enjoys some robustness properties conspicuous by their absence in similar schemes.

For both controllers, we have made the assumption that the robot dynamics can be neglected, hence, the behavior of the overall system is described by the kinematic model (2). Current research is under way to extend our results to consider the robot dynamics.

As clearly illustrated in this paper and thoroughly discussed in [1], [12], I\&I and nonlinear PI constitute a major departure from standard adaptive control techniques, where certainty equivalence parameterized controllers and separable Lyapunov functions are typically utilized. While in I\&I we still preserve the parameterization perspective, but abandon the certainty equivalence and employ Lyapunov functions that mix the plant and the estimator states, in nonlinear PI we propose a simple nonlinear PI structure that gener- ates a stable error equation with a perturbation function that exhibits at least one root. This root is made an attractive equilibrium by suitably adjusting the nonlinear PI gains. It is the authors' belief that, in contrast with other ad hoc controller design techniques, the rigorous mathematical foundation of I\&I and nonlinear PI makes them promising candidates to survive scaling up to more complicated examples.

\section{REFERENCES}

[1] A. Astolfi and R. Ortega, "Immersion and invariance (I\&I): A new tool in nonlinear control design," presented at the Eur. Control Conf. ECC 2001, Porto, Portugal, Sept. 4-7, 2001.

[2] R. Kelly, "Robust asymptotically stable visual servoing of planar robots," IEEE Trans. Robot. Automat., vol. 12, pp. 759-766, Oct. 1996.

[3] E. Coste-Manière, P. Couvignou, and P. K. Khosla, "Visual servoing in the task-function framework: A contour following task," J. Intell. Robot. Syst., vol. 12, pp. 1-21, 1995.

[4] B. E. Bishop and M. W. Spong, "Adaptive calibration and control of 2D monocular visual servo systems," presented at the IFAC Symp. Robot Control, Nantes, France, Sept. 3-5, 1997.

[5] W. E. Dixon, D. M. Dawson, E. Zergeroglu, and A. Behal, "Adaptive tracking control of a wheeled mobile robot via an uncalibrated camera sytem," IEEE Trans. Syst., Man Cybern. B, vol. 31, pp. 341-352, June 2001.

[6] S. Hutchinson, G. D. Hager, and P. I. Corke, "A tutorial on visual servo control," IEEE Trans. Robot. Automat., vol. 12, pp. 651-670, Oct. 1996.

[7] L. Hsu and P. Aquino, "Adaptive visual tracking with uncertain manipulator dynamics and uncalibrated camera," in Proc. 38th Conf. Decision and Control, Phoenix, AZ, Dec. 1999, pp. 1248-1253.

[8] L. Hsu, R. R. Costa, and P. Aquino, "Stable adaptive visual servoing for moving targets," in Proc. of American Control Conf. (ACC), Chicago, IL, June 2000.

[9] R. Kelly, P. Shirkey, and M. W. Spong, "Fixed camera visual servo control for planar robots," in Proc. IEEE Int. Conf. Robotics and Automation, vol. 3, Minneapolis, MN, Apr. 1996, pp. 2643-2649.

[10] H. K. Khalil, Nonlinear Systems. Englewood Cliffs, NJ: Prentice-Hall, 1996.

[11] E. Lefebre, R. Kelly, R. Ortega, and H. Nijmeijer, "On adaptive calibration for visual servoing," in IFAC Symp. Nonlinear Control Systems Design, Enschede, The Netherlands, 1998, pp. 1-3.

[12] R. Ortega and A. Astolfi, "Nonlinear PI control of uncertain systems: An alternative to parameter adaptation," presented at the IEEE Conf. on Decision and Control, Orlando, FL, Dec. 4-7, 2001.

[13] S. Sastry and M. Bodson, Adaptive Control: Stability, Convergence and Robustness. Englewood Cliffs, NJ: Prentice-Hall, 1989.

[14] A. J. Koivo and N. Houshangi, "Real-time vision feedback for servoing of a robotic manipulator with self-tuning controller," IEEE Trans. Syst., Man, Cybern., vol. 21, pp. 134-142, Jan.-Feb. 1991.

[15] J. T. Feddema and C. S. G. Lee, "Adaptive image feature prediction and control for visual tracking with a hand-eye coordinated camera," IEEE Trans. Syst., Man, Cybern., vol. 20, pp. 1172-1183, Sept.-Oct. 1990.

[16] N. P. Papanikolopoulos and P. K. Khosla, "Adaptive robotic visual tracking: Theory and experiments," IEEE Trans. Automat. Contr., vol. 38, pp. 429-445, Mar. 1993. 